\title{
A Metric of Planar Self-Similar Forms
}

\author{
Atsushi IMIYA, Yasumitsu FUJIWAR, Toshiyuki KAWASHIMA \\ Dept. of Information and Computer Sciences, Chiba University \\ 1-33 Yayoi-cho, Inage-ku, Chiba 263, JAPAN \\ imiya@ics.tj.chiba-u.ac.jp
}

\begin{abstract}
In this paper, we define a metric of planar self-similar forms. Self-similarity is one of the fundamental geometric properties which define configurations of geometric elements on the planes and in the space, such as line segments, parts of curves, and blocks. Thus, as the first step in the discrimination of complex objects, which are constructed from basic elements by their structures, we define a metric of self-similarity forms. The iterative function system, IFS, is a method for describing selfsimilar forms. Since a set of metrices defines an IFS, we define a metric arnong self-similar forms using the matrix norm of metrices which define IFS's. We also introduce a method for the estimation of parameters of IFS's from measured data of planar trees.
\end{abstract}

Key words Self-similar forms, Iterative function system, Botanical trees, Distance measure

\section{Introduction}

Syntactic pattern recognition is based on syntactic and symbolic representation of patterns and recognition algorithms [1]. Syntactic and symbolic expression of patterns is suitable for the expression of their topological properties. However, this method is sometimes insufficient for the description of geometric properties. Thus, a syntactic theory which expresses geometric properties is desired [2].

Objects are constructed from elements using a grammar which determines their configurations. Pattern recognition provides metrics for the discrimination of planar figures and time varying signals. These discrimination methods are based on the theory of Hilbert space. In the Hilbert space framework of pattern recognition, a pattern is considered as a point in an abstract space. However, this embedding is inadequate for the recognition of complex geometric shapes which are constructed from elements using a grammar which defines geometric and topological structures. For the definition of metrics among complex objects constructed from elements, a metric among grammars which describe geometric and topological structures of objects is desired. If an object is made up from parts which are similar to whole, the object is self-similar. This is a fundamental property of botanical trees and grasses. Self-similarity is one of fundamental geometric properties which define configurations of elements. Thus, as the first step in the discrimination of complex objects, which are constructed from basic elements, by their structures, we define a metric among self-similar forms. 
The iterative function system, IFS [3] defines a method for the description of self-similar forms in Euclidean space. In Euclidean space, a set of affine transforms defines an IFS. Thus, this set of transformations defines a grammar of the configurations of elements of an object. An affine transformation is defined by a matrix if we adopt homogeneous coordinates for the expression of positions of points in Euclidean space. In this paper, we define a metric among self-similar forms using the matrix norm of affine transformations which defines an IFS. The Housdorff metric directly compares shapes themselves. However, if shapes are expressed by several parameters, the computation cost for the discrimination becomes cheap and results of computation are stable. We also introduce a method for the estimation of parameters of IFS from measured data of planar trees, which is considered as the estimation of grammar for description of complex objects.

\section{Metric of Affine Transformations}

Let $\mathbf{R}^{2}$ be 2-dimensional Euclidean space. Furthermore, define orthogonal coordinate systems $x-y$ in $\mathbf{R}^{2}$. Vectors in $\mathbf{R}^{2}$ are expressed by $\boldsymbol{x}=(x, y)^{T}$ where ${ }^{T}$ indicates the transpose of a vector. The inner product of vectors is defined by $\boldsymbol{x}^{T} \boldsymbol{y}$ and the distances between $\boldsymbol{x}$ and $\boldsymbol{y}$ by

$$
|\boldsymbol{x}-\boldsymbol{y}|=\sqrt{(\boldsymbol{x}-\boldsymbol{y})^{T}(\boldsymbol{x}-\boldsymbol{y})} .
$$

Furthermore, we denote the determinant of an $N \times N$ real matrix $\boldsymbol{A}$ by $|\boldsymbol{A}|$.

For $N \times N$ matrices $\boldsymbol{A}$ and $\boldsymbol{B}$, the matrix inner product is defined by

$$
(\boldsymbol{A}, \boldsymbol{B})=\sum_{m=1}^{N} \sum_{n=1}^{N} a_{m n} b_{m n},
$$

where $a_{m n}$ and $b_{m n}$ are the $m n$-th elements of $\boldsymbol{A}$ and $\boldsymbol{B}$, respectively. Furthermore, the matrix norm is defined by

$$
\|\boldsymbol{A}\|=\sqrt{(\boldsymbol{A}, \boldsymbol{A})} .
$$

Setting $\boldsymbol{A}$ and $\boldsymbol{a}$ to be a $2 \times 2$ matrix and a two-dimensional vector, respectively, an affine transformation on $\boldsymbol{A}$ is expressed by

$$
\boldsymbol{y}=\boldsymbol{A x}+\boldsymbol{a},
$$

where $\boldsymbol{A}$ is invertible. By setting $\boldsymbol{\xi}=\left(x_{1}, y_{1}, 1\right)^{T}$ and $\boldsymbol{\eta}=\left(x_{2}, y_{2}, 1\right)^{T}$ to be the homogeneous coordinates of $\boldsymbol{x}=\left(x_{1}, y_{1}\right)^{T}$ and $\boldsymbol{y}=\left(x_{2}, y_{2}\right)^{T}$, respectively, eq. (4) is expressed as

$$
\boldsymbol{\eta}=\boldsymbol{T} \xi, \boldsymbol{T}=\left(\begin{array}{c}
\boldsymbol{A}, \boldsymbol{a} \\
\boldsymbol{o}^{T}, 1
\end{array}\right)
$$

where $\boldsymbol{o}=(0,0)^{T}$. Thus, a transformation given by eq. (4) is defined by a $3 \times 3$ matrix. In this paper, we also deal with the case that matrix $\boldsymbol{A}$ is singular. We call transformations general affine if we do not demand them to be invertible. Here, we define a distance metric among general affine transformations. 
Definition 1. For two general affine transformations $\boldsymbol{T}_{1}$ and $\boldsymbol{T}_{2}$, we define a distance among transformations as

$$
d\left(\boldsymbol{T}_{1}, \boldsymbol{T}_{2}\right)=\left\|\boldsymbol{T}_{1}-\boldsymbol{T}_{2}\right\| .
$$

Element of a set of general affine transformations are separated into three categories,

$$
\begin{aligned}
C_{+1} & =\left\{\boldsymbol{T}_{i}|| \boldsymbol{T}_{i} \mid>0\right\}, \\
C_{0} & =\left\{\boldsymbol{T}_{i}|| \boldsymbol{T}_{i} \mid=0\right\},
\end{aligned}
$$

and

$$
C_{-1}=\left\{\boldsymbol{T}_{i}|| \boldsymbol{T}_{i} \mid<0\right\},
$$

such that

$$
\left\{\boldsymbol{T}_{i}\right\}_{i=\mathbf{1}}^{n}=C_{+1} \bigcup C_{0} \bigcup C_{-1},
$$

and

$$
C_{a} \bigcap C_{b}=\emptyset, a, b \in T
$$

where $T=\{+1,0,-1\}$. From the definition of the $3 \times 3$ matrix $\boldsymbol{T}$, the sign of the determinant of $\boldsymbol{T}$ is equivalent to that of $2 \times 2$ matrix $\boldsymbol{A}$.

\section{Metric of Iterative Function Systems}

In the following, we deal with the case

$$
\sharp C_{+1}=\sharp C_{0}=\sharp C_{-1}=1,
$$

where $\sharp A$ is the number of elements of set $A$. Furthermore, we write the elements of $C_{\sigma}$ as $\boldsymbol{T}_{(\sigma)}$ for $\sigma \in T$. By setting

$$
s_{\sigma}(\mathbf{F})=\left\{\boldsymbol{\eta} \mid \boldsymbol{\eta}=\boldsymbol{T}_{\sigma} \boldsymbol{\xi}, \boldsymbol{x} \in \mathbf{F}\right\}
$$

for a shape $\mathbf{F}$ and general affine transformations, we obtain the following proposition on $\mathbf{R}^{2}$.

Proposition 2. For vectors in a closed compact set on $\mathbf{R}^{2}$, if each mapping $s_{\sigma}$ satisfies the relation

$$
\left|s_{\sigma}(\boldsymbol{x})-s_{\sigma}(\boldsymbol{y})\right| \leq c_{\sigma}|\boldsymbol{x}-\boldsymbol{y}|, 0<c_{\sigma}<1,
$$

there exists a unique nonempty compact set $\mathbf{F}$ such that

$$
\mathbf{F}=\bigcup_{\sigma \in T} s_{\sigma}(\mathbf{F})
$$

Moreover, if we define a transformation s on nonempty compact set $\mathbf{G}$ as

$$
s(\mathbf{G})=\bigcup_{\sigma \in T} s_{\sigma}(\mathbf{G}),
$$


then

$$
\mathbf{F}=\bigcap_{k=0}^{\infty} s^{k}(\mathbf{G}),
$$

where $s^{0}(\mathbf{G})=\mathbf{G}$ and $s^{k}(\mathbf{G})=s\left(s^{k-1}(\mathbf{G})\right.$ ) [from theorem 9.1 in ref. 5].

We call a fixed point of eq. (15) a self-similar form. Furthermore, eqs. (16) and (17) define an algorithm which generates a self-similar form. Thus, the proposition defines a self-similar form and an algorithm for the generation of them. We call $\mathbf{G}$ the generator of $\mathbf{F}$. In applications, for an appropriate large $n$, we approximate $\mathbf{F}$ by

$$
\mathbf{F}^{n}=\bigcap_{k=0}^{n} s^{k}(\mathbf{G})
$$

A set of general affine transformations $\{\boldsymbol{T}\}_{\sigma \in T}$ defines a self-similar form. Here, we call a set of mappings $\left\{s_{\sigma}\right\}_{\sigma \in T}$ the IFS of $\mathbf{F}$. The IFS's are not restricted to general affine transformations. This paper, however, deals only with a set of general affine transformations.

By using the metric of general affine transformations, we define a metric of self-similar forms.

Definition 3. The metric of two self-similar forms $\mathbf{F}_{1}$ and $\mathbf{F}_{2}$ is defined by

$$
D\left(\mathbf{F}_{1}, \mathbf{F}_{2}\right)=\sum_{\sigma \in T} d\left(\boldsymbol{T}_{(\sigma) 1}, \boldsymbol{T}_{(\sigma) 2}\right),
$$

where $\left\{\boldsymbol{T}_{(\sigma) i}\right\}_{\sigma \in T}$, is a set of general affine transformations which defines $\mathbf{F}_{i}$ for $i=1,2$. .

Since, for each $\sigma \in T, d\left(\boldsymbol{T}_{(\sigma) 1}, \boldsymbol{T}_{(\sigma) 2}\right)$ satisfies the axioms of distance, we obtain the following theorem.

Theorem 4. Equation (19) defines a distance measure.

\section{Parameters of Trees}

As pointed out in reference [5], binary branching on a plane approximates the branching geometry of botanical trees very well. Binary branching is defined by a segment and a pair of subsegments which branch off a segment. According to the biological observation of trees, the binary branching geometry of botanical trees is asymmetric. Asymmetry of the branching geometry of botanical trees is explained from viewpoints of biological functions and the laws of mechanics. If the diameter of one branch is larger than that of the other at a branching point, we observe large amounts of flow of organic liquid and water in the larger branch. Furthermore, if the branching angle of one branch is smaller than that of the other and its length is longer than that of the other, this branch can support 
more descendant branches and leaves than the other. We call the longer branch the leader segment, and we call the other segment the subtending segment.

Let $j$ be the generation of branching and $k, m$, and $n$ be the addresses of segments such that $1 \leq j, 1 \leq k \leq 2^{j-1}$, and $1 \leq m, n \leq 2^{j}$. Then, by setting $\boldsymbol{t}_{j, k}, \boldsymbol{s}_{j+1, m}$, and $\boldsymbol{l}_{j+1, n}$ to be a segment, a subtending segment, and a leader segment, respectively, the binary branching geometry is locally determined by a triple of segments $\left(\boldsymbol{t}_{j, k}, \boldsymbol{s}_{j+1, m}, \boldsymbol{l}_{j+1, n}\right)$ which are connected at a branching point.

We can assume that the pair of branching angles of a tree,

$$
\alpha=\cos ^{-1} \frac{\boldsymbol{t}_{j, k}^{T} \boldsymbol{l}_{j+1, m}}{\left|\boldsymbol{t}_{j, k}\right|\left|\boldsymbol{l}_{j+1, m}\right|}, \beta=\cos ^{-1} \frac{\boldsymbol{t}_{j, k}^{T} s_{j+1, m}}{\left|\boldsymbol{t}_{j, k}\right|\left|\boldsymbol{s}_{j+1, m}\right|},
$$

are constant $[4,5]$. We call $\alpha$ and $\beta$ the leader angle and the subtending angle, respectively. Furthermore, we can assume that the branching ratio $\mu=$ $\left|\boldsymbol{s}_{j, m}\right| /\left|\boldsymbol{l}_{j, m}\right|$ and $r=\left|\boldsymbol{l}_{j, m^{\prime}}\right| /\left|\boldsymbol{l}_{j+1, m}\right|$ are constant $[4,5]$. Here, both $\boldsymbol{\mu}$ and $r$ are positive values smaller the 1 .

There is a unique sequence of the leader segments:

$$
t=\left\langle l_{1,1}, l_{2, n}, \cdots, l_{m, n^{\prime}}\right\rangle, \text { s.t. } l_{1,1}=t_{1,1} .
$$

If the left segment is the leader segment at one branching point, the right segment is the leader segment at the next branching point, and $|\alpha|<|\beta|$, the sequence $t$ forms a zigzag line in a space. This zigzag line makes the main axis of a tree $[4,5]$.

The ratio $\mu$ affects the global shape of a tree because it determines the total length of sequences of segments. Conversely, we can estimate $\mu$ from the global shape of a tree. At each branching point the reader segments support more segments than the subtending segments. Thus, we can assume the relation $\mu=S_{j} / L_{j}$, where $S_{j}$ and $L_{j}$ are the total numbers of generations of segments supported by the subtending segment and by the leader segments, respectively. Moreover, by setting

$$
m_{j}=\sum_{\text {for all sequences }} \sharp t_{j, p}(s), \quad n_{j}=\sum_{\text {for all sequences }} \sharp t_{j, p}(l),
$$

where $\sharp t$ is the total number of elements of a sequence $t$, and

$$
t_{j, p}(s)=\left\langle t_{j, p}, s_{j+1, p}, \cdots, t_{n, q}\right\rangle, \quad t_{j, p}(l)=\left\langle t_{j, p}, \boldsymbol{l}_{j+1, p}, \cdots, t_{n, q^{\prime}}\right\rangle
$$

we obtain the relations $S_{j} \cong \log _{2} m_{j}$ and $L_{j} \cong \log _{2} n_{j}$. Thus, to avoid the case of $n_{j}=1$, we redefine $\mu$ as

$$
\mu=\frac{\log _{2}\left(m_{j}+1\right)}{\log _{2}\left(n_{j}+1\right)} .
$$

If a segment has only one subsegment, we call the subsegment the null pair. If a tree has null pairs, we call it an incomplete tree. If a tree is incomplete, $m_{j}$ and $n_{j}$ depend on the positions of branching points. However, by assuming that 
the ratio $m: n$ is almost constant at any branching point, we can rewrite eq. (24) as

$$
\mu=\frac{\log _{2}(m+1)}{\log _{2}(n+1)}
$$

\section{Geometric Properties of the Metric}

Here, we define an IFS which approximates appearances of binary trees using parameters $\alpha, \beta, \mu$, and $r$, which were defined in the previous section. The following IFS approximates properties of Honda's model tree [4,5], which express the geometry of botanical trees according to certain rules. The meaning of parameters of this system are shown in Table 1 . We define

$$
\begin{aligned}
& \boldsymbol{T}_{-1}=r\left(\begin{array}{ccc}
-\cos \alpha, & -\sin \alpha, & 0 \\
-\sin \alpha, & \cos \alpha, & l / r \\
0, & 0, & 1
\end{array}\right) \text {, }
\end{aligned}
$$

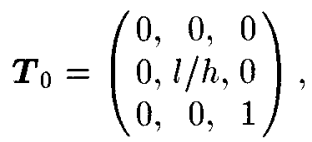

$$
\begin{aligned}
& \boldsymbol{T}_{-1}=\mu r\left(\begin{array}{ccc}
\cos \beta, & -\sin \beta, & 0 \\
\sin \beta, & \cos \beta, & l / \mu r \\
0, & 0, & 1 / \mu r
\end{array}\right) \text {, }
\end{aligned}
$$

and $\mathbf{G}=\left\{(0, l)^{T}\right\}$, where $h=\left(1-r^{2}\right) /(1+r \cos \alpha)$.

We call the segments generated by $\boldsymbol{T}_{-1}$ and the segments generated by $\boldsymbol{T}_{+1}$ the leader branches and subtending branches. Since the determinant of $\boldsymbol{T}_{-1}$ is negative, segments generated by $\boldsymbol{T}_{-1}$ change the sign of the tangent. Thus, the sequences of segments generated by $\boldsymbol{T}_{-1}$ form a zigzag line with the ration $r$. Furthermore, $\boldsymbol{T}_{0}$ defines a line segment since the rank of $\boldsymbol{T}_{\mathbf{0}}$ is one. These line segments determine the total length of branches. Moreover, the segments generated by $\boldsymbol{T}_{+1}$ grow more slowly than branches generated by $\boldsymbol{T}_{-1}$ since $\mu$ is smaller than 1. This means that the sequence of the leader segments forms a zigzag axis which approximates the trunk of a tree. Furthermore, the subtending segments form biuiches of tree. that

By normalizing the length of the first segment $l$ to 1 , for $i=1,2$, we find

$$
l_{1, i}=\left(-r_{i} \sin \alpha_{i}, r_{i} \cos \alpha_{i}+1\right)^{T}
$$

and

$$
s_{1, i}=\left(-r_{i} \mu_{i} \sin \beta_{i},-r_{i} \mu_{i} \cos \beta_{i}+1\right)^{T}
$$

are the ends of the first right branch and the first left branch, respectively. Thus, we obtain the following theorem. 
Theorem 5. If $\alpha_{1} \cong \alpha_{2}, \beta_{1} \cong \beta_{2}$, and $r_{1} \cong r_{2}$,

$$
D\left(\mathbf{F}_{1}, \mathbf{F}_{1}\right)=\sqrt{2}\left(\left|l_{11}-l_{12}\right|+\left|s_{11}-s_{12}\right|\right) .
$$

This theorem implies that the distance measure of trees defined by eqs. (19)-(28) is approximated by the sum of Euclidean distance between the ends of the first branches of the normalized trees if the growth ratios and branch angles of two trees are similar. Figure 1 illustrates the branch geometry of binary trees and the relation of Theorem 5 .

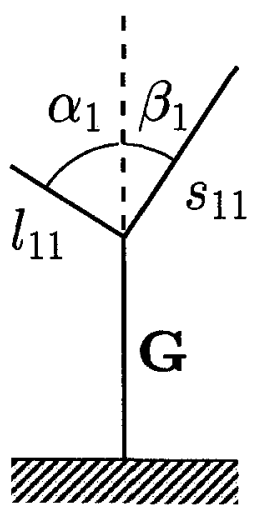

$\mathbf{F}_{1}$

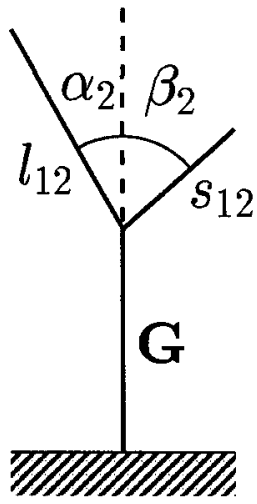

$\mathbf{F}_{2}$

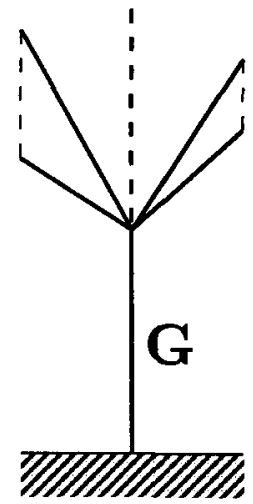

$d\left(\mathbf{F}_{1}, \mathbf{F}_{2}\right)$

Figure 1. Segments of trees and the distances between segments

As shown in Figure 2, this IFS generates binary trees which approximate orthographic views of botanical trees. For the graphical generation of binary trees, the roots of trees are located at the origin of the coordinate system, and the trees grow in the $y$ direction since we set $\mathbf{G}=\left\{(0, l)^{T}\right\}$. The parameters of our IFS are listed in Table 2. Table 3 shows how our metric discriminates these trees.

Table 1.

\begin{tabular}{|r|r|}
$m$ & $:$ number of decendant segments \\
& of the leader branch of a node. \\
$n$ & $:$ number of decendant segments \\
& of the subtending branch of a node. \\
$\mu$ & $:$ branching ratio. \\
$l \quad:$ length of the trunk. \\
$r \quad:$ growth ratio. \\
$\alpha$ & $:$ leader angle. \\
$\beta$ & $:$ subtending angle.
\end{tabular}

Parameters of an iterative function system wich generates binary trees. 


\section{Table 2.}

\begin{tabular}{|c|lccc|}
\hline tree & $m: n$ & $\alpha$ & $\beta$ & $r$ \\
\hline 1 & $0.2: 1.0$ & 10 & -60 & 0.9 \\
2 & $0.5: 1.0$ & 10 & -60 & 0.9 \\
3 & $0.3: 1.0$ & 20 & -40 & 0.9 \\
4 & $0.6: 1.0$ & 20 & -40 & 0.9 \\
5 & $1.0: 1.0$ & 20 & -40 & 0.9 \\
\hline
\end{tabular}

Parameters of iterative function systems which generate the five trees of figure 2. The angles are expressed in degree.

Table 3.

\begin{tabular}{c|ccccc} 
& 1 & 2 & 3 & 4 & 5 \\
\hline 1 & 0 & 0.03 & 0.94 & 1.19 & 1.74 \\
2 & & 0 & 0.95 & 1.17 & 1.72 \\
3 & & & 0 & 0.56 & 1.20 \\
4 & & & & 0 & 0.64 \\
5 & & & & & 0
\end{tabular}

Distance matrix of trees in figure 2. Since the distance matric is symmetric, only the entries of the upper triangle are listed.

\section{Conclusions}

In this paper, we discussed the identification, discrimination, description, and classification of patterns in the same context by focusing our attention on selfsimilar forms. Furthermore, our method will provide possibility for studying recognition and representation in the same context, since self-similarity provides a fundamental computational method for the generation of complex patterns in computer graphics. Moreover, the metric may anable us to quantitatively classify the performance of algorithms of computer graphics which generate artificial forms.

The first author expresses his thanks to Professor H. Hirata of Chiba University who introduced him to the book by N. MacDonald. Part of this research is based on the undergraduate project of Y. Fujiwara at Department of Information and Computer Sciences, Chiba University. 
1

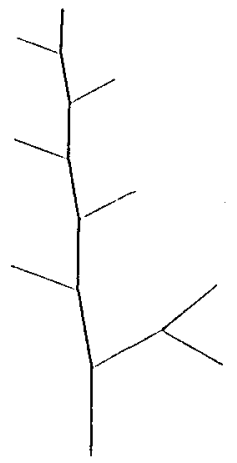

3

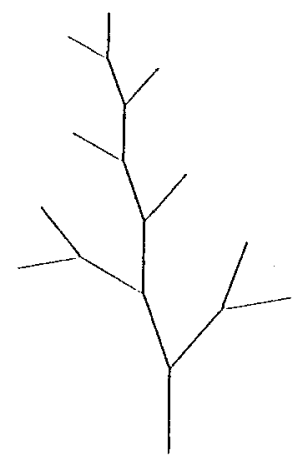

2

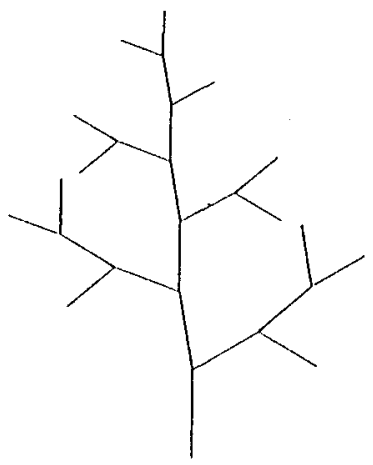

4

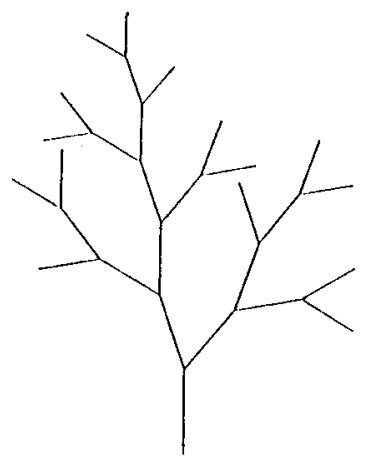

5

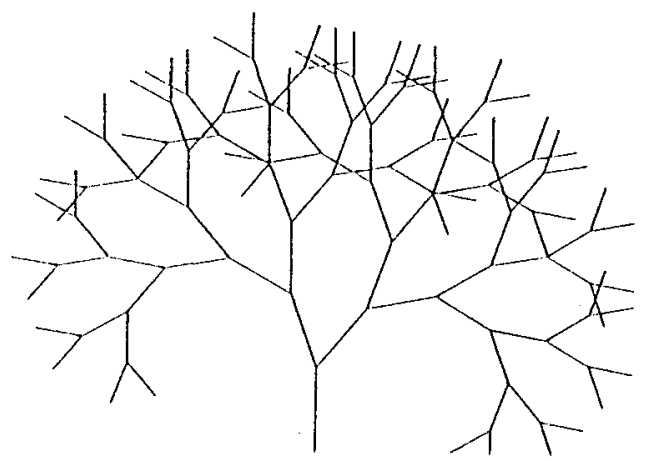

Figure 2. Five trees which are generated using an iterative function system. The parameters for the generation of these trees are listed in Table 2. Distances among these five trees are listed in Table 3 in the form of the comparison matrix. 


\section{References}

[1] Fu, K.S., Syntactic Method in Pattern Recognition, Academic Press; New York, (1974).

[2] Grenader, U., General Pattern Theory, Oxford University Press; Oxford, (1993).

[3] Falconer, K., Fractal Geometry: Mathematical Foundations and Applications, John-Wiley \& Sons; Chichster, (1990).

[4] Honda, H., Tomlinson, P.B., and Fisher, J. B., Computer simulation of branch interaction and regulation by unequal flow rates in botanical trees, American Journal of Botany, 69, (1918) pp.569-585.

[5] MacDonald, N., Trees and Networks in Biological Models, John-Wiley \& Sons; Chichster, (1983). 